\title{
EFFEI DU PLASMON DE SURFACE LOCALISÉ sur les propriétés des sources organiques (OLED)
}

\author{
Samira KHADIR* \\ Mahmoud CHAKAROUN \\ Azzedine BOUDRIOUA \\ Université Paris 13, Sorbonne \\ Paris Cité, Laboratoire de Physique \\ des Lasers, CNRS (UMR7538), \\ 93430 Villetaneuse, France. \\ * Actuellement à : Institut \\ Fresnel, Faculté des Sciences \\ de Saint Jérôme, avenue \\ Escadrille Normandie-Niemen, \\ 13397 Marseille
}

\section{L}

a technique d'évaporation thermique est largement utilisée pour la fabrication de NP aléatoires (RMN, random metallic nanoparticles) en raison des avantages technologiques liés à la facilité de sa mise en œuvre et son adaptation pour la fabrication de dispositifs sur de grandes surfaces. Elle permet de déposer des NP dans n'importe quelle position à l'intérieur de l'hétéro-structure OLED et de contrôler leur distance par rapport à la couche émissive. La taille des NP est obtenue en variant l'épaisseur de la couche déposée qui s'agglomère sous forme d'agrégats pour des épaisseurs inférieures à quelques nanomètres. En revanche, cette technique ne permet pas de contrôler la périodicité et la taille des nano-objets. Cependant, la distribution aléatoire des NP peut être exploitée pour amplifier les propriétés optiques des entités situées dans l'environnement proche des NP. En effet, les NP de petite taille se comportent comme de bons résonateurs, permettant un fort confinement du champélectromagnétique, et celles de grande taille se comportent comme des nano-antennes qui couplent les modes plasmon à l'extérieur des NP. Cette situation peut induire la

\section{L'utilisation de l'effet plasmonique de nanoparticules} métalliques (NP) est l'une des voies les plus prometteuses pour améliorer les performances optiques et électriques des diodes électroluminescentes organiques (OLED). Le choix du métal, la taille et la position des NP sont liés aux propriétés physiques recherchées mais aussi aux approches et aux techniques permettant leur fabrication. Deux approches peuvent être utilisées : random metallic nanoparticules (RMN) et periodic metallic nanoparticules (PMN).

formation de points chauds (hot spots) entre les NP [1]. En raison des avantages cités précédemment, on trouve dans la littérature, plusieurs travaux récents dédiés à l'amélioration du rendement des OLED par l'utilisation des structures RMN.

Concernant les NP de type PMN, elles peuvent être fabriquées par voie électrochimique, par lithographie à nano-sphère, par nano-impression ou par lithographie électronique. Ces techniques different en termes de facilité de réalisation, de surface, de temps de fabrication et de la qualité des NP. Dans le cas des OLED plasmoniques, ce type de structures présente un autre inconvénient lié à la maîtrise de leur position à l'intérieur de l'hétéro-structure organique (l'OLED) du fait que la structure PMN doit être fabriquée au préalable sur des substrats conducteurs et qui résistent aux solvants (ITO par exemple) [2]. Cependant, les PMN ont fait l'objet de plusieurs études montrant l'effet du plasmon de surface localisé sur les propriétés optiques des émetteurs organiques. En fait, deux types de plasmon peuvent apparaître en utilisant un réseau périodique de NP métalliques [3]. Pour de courtes périodes du réseau (plus petite que la longueur d'onde), seule la résonance du

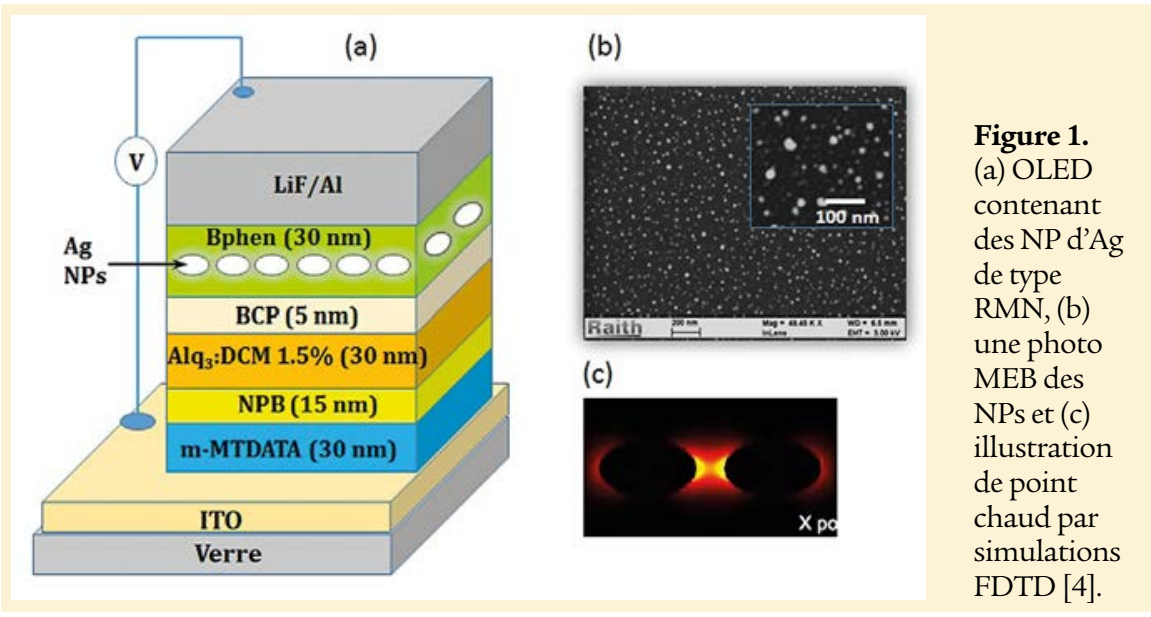


(b)
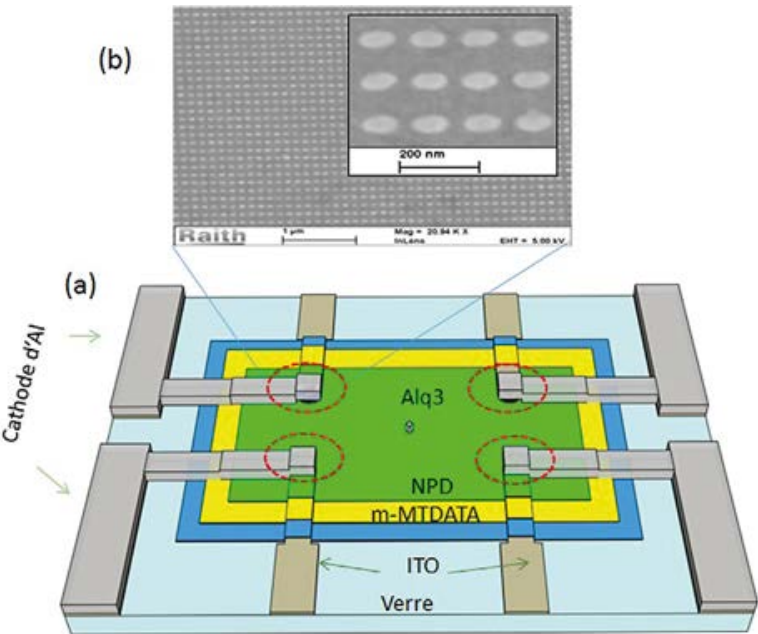

Figure 2. (a) $\mu$-OLED contenant des NP d'Al de type PMN, (b) une photo MEB des NPs PMN [2]. plasmon de surface localisée (LSPR) des NPs peut apparaître. Pour des périodes comparables à la longueur $\mathrm{d}^{\prime}$ onde, en plus du LSPR, d'autres modes peuvent apparaître, appelés modes plasmons du réseau (SLR : surface lattice resonance). Le SLR peut présenter un véritable intérêt pour l'utilisation de structures PMN. De plus, le pic LSPR (spectre d'extinction par exemple) des structures PMN est étroit par rapport à celui des NP métalliques aléatoires (RMN). Ainsi, on peut facilement ajuster la longueur d'onde de résonance des NP pour correspondre au pic d'émission souhaité de l'OLED.
Les travaux récents montrent que la LSPR des NP RMN ou PMN peut mener à un renforcement global des performances del'OLED vial'amélioration de linjection et du transport de charges, l'amélioration du transfert d'énergie dans les systèmes guest-host et la diminution de la durée de vie des excitons entrainant une augmentation de l'électroluminescence et de l'efficacité du dispositif. L'utilisation des NP de type PMN présente des propriétés optiques très intéressantes qui peuvent être exploitées pour améliorer la directivité et la cohérence de l'émission des OLED.

Par ailleurs, l'utilisation d'un système émetteur-NP métalliques dans une microcavité peut donner lieu à une amélioration supplémentaire de l'émission, si la longueur d'onde de la LSPR et le mode de cavité coïncident. Dans ce cas, à notre connaissance, très peu d'études ont combiné l'effet plasmonique et l'effet d'une cavité [4].

\section{POUR EN SAVOIR PLUS}

[1] J.B. Khurgin, G. Sun, Enhancement of optical properties of nanoscaled objects by metal nanoparticles, J. Opt. Soc. Am. B, 26, 83 (2009)

[2] R.F. Garcia, L. Zeng, S. Khadir, M. Chakaroun, A.P. Fischer, A. Boudrioua, Enhanced electroluminescence of organic light emitting diode by localized surface plasmon using Al-periodic structure, J. Opt. Soc. Am. B 33, 246 (2016)

[3] A.D. Humphrey, W.L. Barnes, Plasmonic surface lattice resonances in arrays of metallic nanoparticle dimmers, J. Opt. 18, 035005 (2016)

[4] S. Khadir, M. Chakaroun, A. Belkhir, A. Fischer, O. Lamrous, A. Boudrioua, Localized surface plasmon enhanced emission of organic light emitting diode coupled to DBR-cathode microcavity by using silver nanoclusters, Opt. Express 23, 23647 (2015)

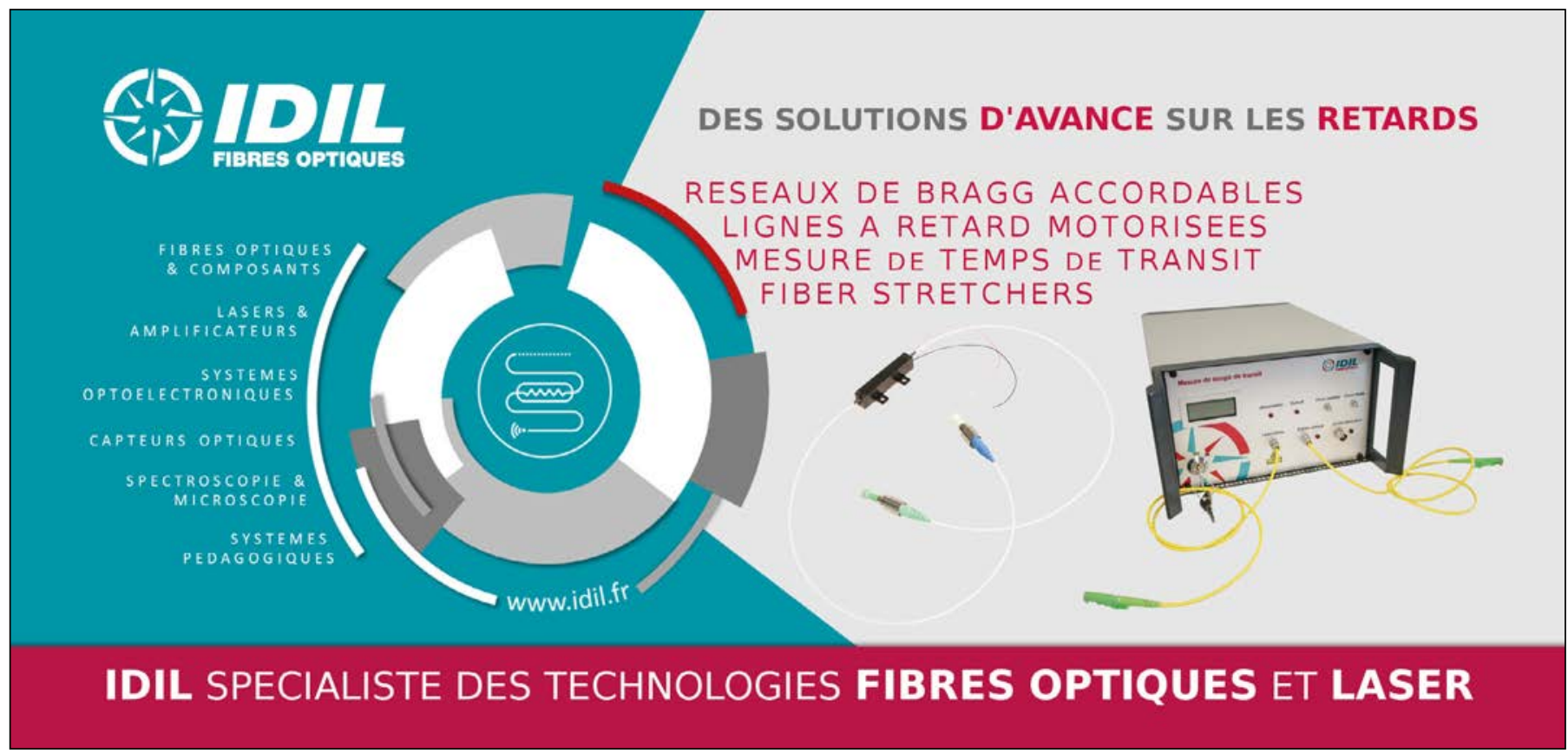

